\title{
Cadena de Valor Turismo de Salud del Área Metropolitana de Cúcuta
}

\author{
Health Tourism Chain Value in the Metropolitan Area in Cucuta
}

\section{Cadeia de Valor Turismo de Saúde da Área Metropolitana de Cucuta}

\author{
Julio Alfonso González-Mendoza ${ }^{1}$, Marlen Del Socorro Fonseca-Vigoya ${ }^{2}$
}

Forma de citar: J. A. González-Mendoza, M. S. Fonseca-Vigoya, "Cadena de Valor Turismo de Salud del Área Metropolitana de Cúcuta”, Respuestas, vol. 21, no. 1, pp. 28-44, 2016.

Recibido:

Abril 30 de 2015

Aceptado:

Julio 16 de 2015

\section{Resumen}

Antecedentes: La economía colombiana, se ha caracterizado por una amplia apertura y libre mercado, tanto que hoy tiene tratados comerciales con los bloques más importantes del mundo. Esta característica exige tener unas condiciones de alta competitividad y diversificación, por lo que los Gobiernos nacional y regional intentan impulsar algunos sectores que tradicionalmente han sido inexplotados como el turismo y específicamente el Turismo de Salud, con el fin de convertirlo en un sector de clase mundial, que coadyuve a generar fuentes de riqueza, empleo y diversificación de la economía. El Turismo Médico es un sector que cada día toma mayor fuerza y credibilidad y en algunos países como Singapur, Malasia o Costa Rica constituyen un aporte importante al PIB. Objetivo: Describir la cadena de valor de sector Turismo de Salud, del Área de Cúcuta, determinar sus ventajas y desventajas y proponer estrategias que permitan su desarrollo como alternativa económica para la zona de frontera. Método: Documental y cuantitativo, enfocado en los actores del subsector, con especial énfasis en las Instituciones Prestadoras de Salud, y teniendo como teorías fundamentales, la Cadena de Valor y el Diamante de Porter. Resultados: La región cuenta con la infraestructura, una posición geoestratégica y las condiciones sociales y económicas necesarias, para lograr el surgimiento del subsector, aunque para lograrlo se requiere, principalmente, que las instituciones de salud logren acreditaciones internacionales, sus trabajadores tengan un buen nivel de bilingüismo y se desarrolle una adecuada estrategia de mercadeo y de articulación entre actores del sistema. Conclusiones: Existen las condiciones necesarias para que el turismo de salud se convierta en una alternativa económica viable, siendo necesario diseñar políticas y estrategias dirigidas a la articulación de los actores, el mejoramiento de la infraestructura, la capacitación del recurso humano y la certificación de calidad de las instituciones prestadoras de salud.

Palabras Clave: Alternativa económica, Cadena de Valor, Norte de Santander, Turismo de Salud.

\section{Abstract}

Background: The Colombian economy has been characterized by a wide open free market, including current agreements with major trade blocs in the world. This feature 
requires high competitiveness and diversification conditions, thus national and regional governments must try to promote some sectors that have traditionally been unexploited such as Health Tourism, in order to make it become a world-class industry that contributes to generate economic wealth, employment and diversification of the economy. Health tourism is a growing industry in countries such as Singapore, Malaysia, and Costa Rica, making an important contribution to the GPD. Objective: To establish the chain value in Health Tourism in the Cúcuta area, determining its advantages and disadvantages and proposing strategies for its development as an economic alternative to the border area. Method: Documental and quantitative, focused on Health Provider Institutions, with chain value and porter diamond as fundamental theories. Results: The results indicate that the region has the necessary infrastructure, a geostrategic position and the social and economic conditions that favor the emergence of the sector. It is necessary for the health institutions to obtain international accreditation, bilingual skills of the employees, and an adequate marketing strategy and articulation between the actors of the system. Conclusions: There are conditions for development of health tourism as a viable economic alternative. Design of policies, infrastructure improvement, human resource training, and quality certification of health institutions are necessary.

Keywords: Economic alternative, Chain Value, Norte de Santander, Health tourism.

\section{Resumo}

Antecedentes: A economia colombiana tem sido caracterizada por um mercado livre aberto, tendo hoje acordos comerciais com grandes blocos do mundo. Esta característica exige ter uma alta competitividade e diversificação, de modo que os governos nacionais e regionais tentam empurrar alguns setores que tradicionalmente tem sido inexplorado, como o turismo e mais especificamente o Turismo de Saúde, a fim de torná-lo uma indústria de classe mundial, que contribua para a geração de riqueza, emprego e diversificação da economia. O Turismo Médico é um setor que cada dia toma maior força e credibilidade e em alguns países como Singapura, Malásia ou Costa Rica constitui um aporte importante ao PIB. Objetivo: Descrever a cadeia de valor do setor Turismo de Saúde da área de Cucuta, determinar suas vantagens e desvantagens e propor estratégias que permitam seu desenvolvimento como alternativa económica para a área de fronteira. Método: Documental e quantitativo, enfocado nos atores do subsetor, com particular ênfase nas Instituições Emprestadoras de Saúde, e tendo como teorias fundamentais: a Cadeia de Valor e o Diamante de Porter. Resultados: A região conta com a infraestrutura, uma posição geoestratégica vantajosa e as condições sociais e económicas necessárias para alcançar o surgimento do subsetor, embora para atingi-lo se requer, principalmente, que as instituições de saúde consigam a acreditação internacional, os seus trabalhadores tenham um bom nível de bilinguismo e se desenvolva uma adequada estratégia de marketing e de articulação entre atores do sistema. Conclusões: Existem as condições necessárias para que o turismo de saúde se torne uma alternativa econômica viável, sendo necessário elaborar políticas e estratégias dirigidas à articulação dos atores, melhoramento da infraestrutura, formação de recurso humano e certificação de estratégias de qualidade nas instituições de saúde.

Palavras-chave: Alternativa económica, Cadeia de Valor, Norte de Santander, Turismo de Saúde.
Enero - Junio 2016

ISSN 0122-820X

E-ISSN 2422-5053

PP: 28-44 
No. 1

Enero - Junio 2016 ISSN 0122-820X E-ISSN 2422-5053

PP: 28-44

\section{Introducción}

La economía fronteriza tiene la particularidad que se nutre de las ventajas que le brindan los modelos económicos de ambos países, generando oportunidades de comercio e intercambio y por ende de empleo, así sea de manera informal y transitoria, pero también se ve afectada por las dificultades que pueden tener las dos orillas; es decir la economía de frontera es volátil e inestable dependiendo de las condiciones económicas de los dos países.

Por ello los gobiernos nacional, departamental y municipal intentan crear estrategias que posibiliten la diversificación de la economía y disminuyan la dependencia e impacto de las fluctuaciones de la economía venezolana. Uno de esos proyectos es el desarrollo y consolidación del Turismo de Salud, que impulsaría otras cadenas productivas como transporte, empresas farmacéuticas, hoteles, restaurantes y comercio, estimulando la creación de nuevos negocios que diversifiquen la economía y a la vez soporten al sector.

El objetivo de este trabajo es describir la cadena de valor del subsector de Turismo de Salud en el Área Metropolitana de Cúcuta, identificar los principales actores y sus interacciones, apoyados en las teorías de Centro-Periferia y Ventaja Competitiva de Porter, para lo cual se estudiaron 127 Instituciones Prestadoras de salud, que junto con la información recabada de otros actores que conforman el subsector, permite mostrar sus potencialidades y las debilidades.

Los principales hallazgos indican que el subsector es una alternativa viable para esta zona de frontera, aunque haya deficiencias en infraestructura, tecnología, capacitación de recurso humano, nivel de bilingüismo, certificación de los servicios ante entidades del orden internacional $y$ políticas $o$ instrumentos que articulen los esfuerzos de los actores. La mayoría de las especialidades solicitadas por los turistas médicos, se prestan en instituciones de salud de la región. Los clientes potenciales provienen principalmente de Estados Unidos, Canadá, Europa, países del Caribe y Venezuela; estos últimos países están muy cerca de Norte de Santander, que le concede una posición geoestratégica privilegiada y la oportunidad de ofrecer los servicios de salud sin las barreras idiomáticas.

La venta de los servicios a pacientes de otros países, se basa principalmente en el diferencial de precios, que para Colombia pueden representar un ahorro entre el 10\% y el $40 \%$, y si además se explota adecuada y articuladamente un "esencialismo estratégico", es decir la belleza natural de los paisajes, riqueza histórica, diversidad cultural, y posición estratégica de la región, puede encontrarse una alternativa económica viable para Norte de Santander.

\section{Materiales y Métodos}

La investigación se realizó con dos metodologías cuantitativa y documental. Con el método cuantitativo se aplicaron encuestas a instituciones prestadoras de salud del Área Metropolitana de Cúcuta, Norte de Santander, Colombia, en donde según la Superintendencia Nacional de Salud [1], se encuentran instaladas 168 instituciones prestadoras de salud. Si se asumen un margen de error del 5\%, una desviación estándar de 1.96, utilizando el paquete estadístico STAT, se obtiene una muestra de 117 IPS, en las cuales se aplicó el estudio.

El método documental, además de la consulta de las principales bases de datos de investigación científica y la recopilación y análisis de la información, se estudió la normativa y estadística de Colombia, Norte de Santander y del Área Metropolitana de Cúcuta acerca del tema tratado. Algunos datos adicionales se solicitaron directamente a entidades como el Instituto Departamental de Salud, Cotelco, instituciones prestadoras de salud, Instituto Colombiano de Normas 
Técnicas (ICONTEC), Cámara de Comercio de Cúcuta, Cámara de Comercio de Medellín. La información analizada se estructuró conforme a las teorías de Las Cinco Fuerzas y Cadena de Valor de Michael Porter, lo cual permite contextualizar y comprender adecuadamente la situación del sector.

\section{Resultados y Análisis}

Turismo de Salud también conocido como turismo médico o asistencia sanitaria global, es un término acuñado inicialmente por las agencias de viajes y los medios de comunicación para describir la práctica de viajar a través de las fronteras internacionales y obtener atención de salud [2]. Turismo es el proceso de salir de casa a buscar tratamiento y cuidado médico en el extranjero o en otro lugar del país [3], en algunos casos, el turismo médico incluye esparcimiento y vacaciones para los pacientes, sus familiares y acompañantes.

La práctica de turismo médico, pese a ser un término acuñado recientemente, se remonta a los orígenes del hombre, que viajaba en busca de alivio a sus enfermedades o descanso de la cotidianidad. Los antiguos griegos y egipcios, por ejemplo, buscaban aguas termales las cuales daban mejoría a su salud, recreación y esparcimiento; los sumerios, hacia los años 4000 A.C, construían complejos turísticos, alrededor de fuentes de agua, preferiblemente termales, acompañados de majestuosos templos que seducían, no solo a pacientes sino a turistas en general [4]. Tal vez fueron los romanos quienes concibieron el concepto de turismo de salud, similar al que se conoce hoy, utilizaron la bondad de las aguas termales $\mathrm{y}$ alrededor construyeron sitios en los que ofrecían servicios como masajes, unción con aceite y lugares de descanso [5].

Antes del fenómeno de globalización, los habitantes de países con bajos y medianos ingresos se trasladaban a los países industrializados en busca de atención especializada; en la actualidad, muchos de los países del primer grupo se han hecho famosos por su buena atención médica, tratamiento de enfermedades ofrecidos a costos mucho más reducidos que aquellos que ofrecen los países de origen de los turistas [6].

En el mundo moderno, la búsqueda de asistencia médica fuera de las fronteras es una práctica que gana importancia, principalmente debido al auge de las comunicaciones y la globalización, que han facilitado conocer sitios nuevos, exóticos, con altos niveles tecnológicos y de fácil acceso, que brindan privacidad, comodidad y precio bajos y ofrecen mejoría a las enfermedades, esparcimiento a su rutina, alivio al estrés y la oportunidad de conocer y compartir otras culturas.

Son diversas las teorías que se ha intentado aplicar al turismo de salud, entre ellas la del postcolonialismo, definido como [7], una corriente que estudia la construcción y la expresión de una identidad propia de cada país o región. Según esta teoría, los lugares donde se brinda el servicio de salud, están conformados por el atractivo y eficiencia de los aspectos físicos del lugar, infraestructura, condiciones sociales y culturales de los pobladores, que combinados entre sí establecen un ambiente adecuado y diferente para lograr bienestar y salud al paciente [8]. Los entornos terapéuticos comprenden dos elementos: a) internos conformados por la naturaleza, los construidos por el hombre y las actividades y diarias relacionadas con el servicio; y b) los externos que comprenden valores, creencias, relaciones sociales y nociones de territorialidad [9].

Los escenarios construidos con el propósito de prestar servicios de salud a los turistas, combinan los elementos internos y externos, para ser atractivos y capaces de trascender, a otras sociedades, conservando su propia identidad y brindando al cliente el servicio
Enero - Junio 2016 ISSN 0122-820X E-ISSN 2422-5053 PP: 28-44 
No. 1

Enero - Junio 2016 ISSN 0122-820X E-ISSN 2422-5053

PP: 28-44 deseado, con calidad igual o mejor a la que se pueda encontrar en los países desarrollados.

Otra perspectiva de la misma teoría [10], divide el turismo de salud en tres categorías. La primera tiene como eje la explotación de lugares que han desarrollado y sostenido cierta reputación, basados principalmente en la belleza natural de los paisajes y sus contribuciones para encontrar condiciones de salud favorables. En la mayoría de los casos, estos lugares tienen aguas termales o fuentes de agua como recurso primordial de la existencia de la vida, añadiendo a ésta, un significado espiritual.

El segundo grupo se centra en las redes menos formales que incorporan en su atención tradiciones biomédicas. Por ejemplo incluyen medicina alternativa, spa, yoga, tai chi, cafeterías vegetarianas y jardines, como aspectos complementarios del paquete de turismo médico [11]. En este grupo se encontrarían las prácticas médicas de los indígenas o aldeanos. El tercer grupo son espacios que giran en torno intereses particulares y culturales, basados en valores sociales, donde sexualidad, raza, género, clase social, grupos étnicos, adulto mayor, etc. son elementos primordiales para la especialización de la atención en salud.

La venta de servicios de los países desarrollados, hasta ahora se ha basado principalmente en la diferencia de precios [12], aunque esa estrategia puede cambiar, incluyendo dentro de la negociación, el esencialismo estratégico, es decir ofertando los aspectos fundamentales y diferenciadores de su cultura, religión, paisajes y ubicación considerándolas como ventaja competitiva [13].

Sin embargo esta relación comercial, puede generar problemas como inequidad en la atención entre los pobladores locales, que tal vez no cuentan con suficiente cantidad de dinero para solicitar un servicio de alta

calidad, contrario a los turistas que sí la tienen, por lo que es necesarios establecer relaciones de equidad y respeto, dando equilibrio entre la atención del extranjero que deja recursos necesarios para el desarrollo económico, sin menoscabo de la dignidad, el respeto y la salud de los pobladores locales [14] y evitar choques culturales, por ejemplo, que una enfermera de la periferia se sienta intimidada por las acciones del paciente de centro [15].

Otro cuerpo teórico que explica el turismo de salud, y en general sobre los sectores económicos, es la Ventaja Competitiva [16], que plantea que existen dos determinantes fundamentales, para el análisis estructural de las industrias y por ende de una competitividad sustentable. El primer determinante, consiste en el conocimiento de las reglas de la competencia y la forma de influir en ellas a su favor. Estas reglas provienen del contenido y conocimiento de cinco fuerzas de competencia: a) la entrada de más competidores, b) la amenaza de los sustitutos, c) el poder negociador de los compradores, d) el poder de negociador de los proveedores y e) la rivalidad entre los competidores actuales [16]. El conocimiento y gestión de estos factores determina la rentabilidad de la industria debido a su influencia en los precios, los costos y la inversión.

La posición que ocupa la empresa o dentro de la industria, es el segundo determinante, y para ello se requiere observar dos tipos de ventaja competitiva: a) liderazgo de costos, consistente en tener la mayor eficiencia y eficacia en sus recursos, y b) diferenciación, enfocado a dar la mejor calidad del producto al cliente. Estos dos determinantes, combinados con la naturaleza de la empresa o sector dan origen a las estrategias genéricas para un desempeño superior.

A su vez la naturaleza de la industria y el análisis de la ventaja competitiva requiere un medio sistemático de examinar todas 
las actividades que se realizan y la forma en que están articuladas, para ello [16] plantea el concepto de cadena de valor como herramienta básica que permite dividir la industria en actividades relevantes a fin de entender el comportamiento de los costos y las potenciales diferenciales.

Apoyados en las teorías de Ventaja Competitiva, y Cinco Fuerzas de Porter, se contextualiza el sector de turismo médico del área metropolitana de Cúcuta, mostrando sus potencialidades y debilidades, base fundamental para la construcción de estrategias que permitan consolidar una alternativa económica para la región y contribuir al propósito de ser tener un sector de clase mundial.

\subsection{Diamante de Porter}

Según la teoría de ventaja competitiva de Porter, para tener un desempeño superior de la empresa o sector, es necesario conocer las reglas de la competencia y lograr colocarlas a su favor, por tanto se desarrolla en esta parte el diamante de Porter, describiendo las fuerzas que mueven el sector de turismo de salud en la región. (Ver Figura 1).

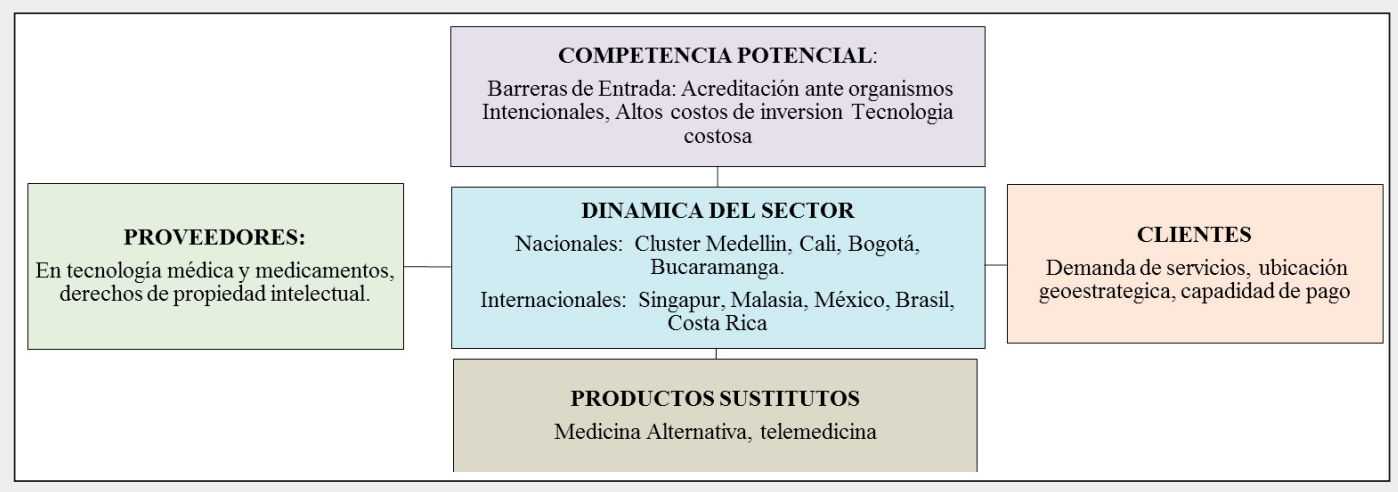

Figura1. Cinco fuerzas de Porter de turismo de salud de área metropolitana de Cúcuta Fuente: Autores

Competencia Potencial: Como el turismo de salud, es un fenómeno generado por un concepto más amplio llamado globalización, el sector salud presenta nuevos desafíos tales como: "la necesidad de contar con estándares para la protección de la salud de las personas en su carácter de consumidoras" [6].

Las instituciones de salud [17], preferidas por los pacientes son aquellas que cuentan con acreditación y reconocimiento de instituciones internacionales como Joint Commission International (JCI), Joint Commission on Accreditation of Healthcare Organization (JCAHO) o la International Organization for Standardization (ISO), organismos de Estados Unidos que acreditan, la dedicación y la excelencia en el servicio y para lograrlo se requiere de una gran inversión en infraestructura, tecnología, recursos humanos calificado y diseño de procesos.

Los estándares de acreditación son un factor vital en la evaluación de la calidad de la atención proporcionado por un hospital extranjero [18]. Los pacientes buscan clínicas y hospitales con estándares internacionales, y los principales referentes en este sentido es la certificación de la $J C I$ que ha sido recibida por más de 635 instituciones a nivel mundial [19]. Colombia cuenta con dos instituciones acreditadas por JCI y 22 más en trámite [16].

Considerando que para establecerse como proveedor en salud se requiere de una alta inversión financiera, altos estándares de calidad exigidos por las normas nacionales, tecnología e infraestructura costosa, personal
Enero - Junio 2016 ISSN 0122-820X E-ISSN 2422-5053 PP: 28-44 
No. 1

Enero - Junio 2016 ISSN 0122-820X E-ISSN 2422-5053

PP: $28-44$ altamente cualificado para desempeñar sus funciones, complejas relaciones entre los actores del sector y especialmente las certificaciones internacionales que debe tener una institución prestadora de salud para que los pacientes al menos la consideren en sus planes. Se considera que las barreras de entrada al sector son altas y por tanto es difícil la entrada de nuevos competidores. Se resalta que además de cumplir estos requisitos, no es garantía de éxito en el negocio sino que es la condición fundamental para intentar atraer los turistas de salud.

Los Clientes: Los clientes o compradores de turismo médico para el Área Metropolitana de Cúcuta, son principalmente los provenientes de Venezuela y países del Caribe, debido a su cercanía y a la facilidad del idioma y la similitud cultural. Son igualmente clientes potenciales los colombianos que viven en Estados Unidos, Canadá y Europa que tienen un alto poder adquisitivo y que buscarían el servicio de salud en esta región no solo para lograr mejoría en su condición, sino para aprovechar el regreso a su tierra y a su esencialismo natural. Otro nicho importante de clientes son los extranjeros que por razones de confidencialidad, precio y oportunidad pueden hacer uno de esta alternativa en salud.

Como se ha dicho, principales razones para que los clientes o compradores internacionales, busquen solución a los problemas de salud en otros países son: a) el precio, factor clave [20]. Un paciente de Estados Unidos, por ejemplo, que recibe tratamiento médico en Tailandia, puede ahorrar hasta un 75\% [21]; b) Muchos procedimientos no son cubiertos por el seguro de salud; c) Largas esperas para la realización de un procedimiento [22], que puede encontrar en forma más oportuna en otro lugar; c) La privacidad especialmente para los pacientes que buscan cirugía plástica, procedimientos de cambio de sexo o tratamientos relacionados con rehabilitación por drogadicción [23].
El principal determinante por el cual los estadounidenses viajan al extranjero para la atención en salud, es el precio [18]. Los procedimientos médicos en Tailandia cuesta sólo un tercio de lo que en Estados Unidos, por tanto el atractivo de un ahorro sustancial motiva la demanda de servicios médicos en los países en desarrollo. Se estima que el costo promedio de los procedimientos médicos en Colombia es de entre el $25 \%$ y el $40 \%$ del costo en Estados Unidos [19].

Considerando que el paciente tiene capacidad de pago y varias alternativas donde se pueda recibir un servicio de calidad, alto nivel de confianza y precio atractivos, se puede concluir que el poder de negociación de los clientes es alto, ya que éste podría pagar un poco más, si fuera necesario, para encontrar una adecuada atención que satisfaga sus necesidades en salud [24].

Los Proveedores del sector son los fabricantes y distribuidores de dispositivos médicos, tecnología y medicamentos. Estos bienes producen una alta sensibilidad en los precios del servicio, debido al monopolio de patentes y a la exclusividad en la explotación de sus innovaciones. Se estima que los precios de medicamentos en Colombia son los más costosos de la región, aunque el Gobierno nacional ha tomado medidas con el fin de equiparar los precios con otros países de la región. El poder de negociación es alto debido a la exclusividad de las patentes y al monopolio de explotación de los laboratorios.

Los Sustitutos: Los tratamientos médicos preferidos por los turistas, van desde medicina preventiva como chequeos y exámenes de salud y procedimientos menores en cirugía estética y dental, hasta procedimientos invasivos mayores como cirugía a corazón abierto, reemplazo de rodilla o cadera, columna vertebral y transformación de género [25]. 
Los productos sustitutos, corresponden a los métodos de curación tradicional, tales como hierbas o acupuntura china, llamados medicina alternativa. Sin embargo, los tratamientos que tienen que ver con los órganos internos del cuerpo, como el corazón, los pulmones y el cerebro, la posibilidad de un producto sustituto son prácticamente nulos [26].

Rivalidad entre los Competidores: El turismo médico es una industria emergente en todo el mundo y tiene como premisa fundamental que el mismo cuidado, o incluso de mejor calidad, pueden estar disponibles en otro país, a un costo más asequible [26]. Esta industria produce, solo en Estados Unidos 60 mil millones de dólares al año y está creciendo a un ritmo del 20\% al año [27].

Los destinos preferidos son Argentina, Brunei, Cuba, Colombia, Costa Rica, Hong Kong, Hungría, India, Jordania, Lituania, Malasia, Filipinas, Singapur, Sudáfrica, Tailandia y, recientemente, Arabia Saudita, Emiratos Árabes Unidos, Corea del Sur, Túnez, Ucrania y Nueva Zelanda [28]. Algunos países consideran el Turismo de Salud como una prioridad económica, así para India será un motor clave de su desarrollo, en Singapur se estima le aportó el 1.1\% en PIB en 2012 [29], mientras que Tailandia espera que este sector aporte el 4\% del PIB [30].

En el 2007, 750.000 estadounidenses viajaron a otros países en búsqueda de tratamientos médicos y estima que este volumen puede llegar a 14 millones de pacientes en 2017 [3]. El aumento del costo de los tratamientos de cuidado de la salud en los Estados Unidos es el mayor motivo de la búsqueda de solución a las necesidades de tratamiento médico en otros países.

Proexport Colombia [20], se estima que en Colombia el turismo de salud es un sector aún incipiente, apenas el $2.2 \%$ de los turistas que ingresan al país son turistas de salud; otros países como México, Costa Rica y Argentina han logrado mayores avances [17]. El turismo de salud en Colombia ha sido desarrollado principalmente en Bogotá, Medellín Barranquilla y Bucaramanga donde existen clínicas de cuarto nivel, con instalaciones y tecnologías modernas y acreditación internacional Joint Commission, que en la actualidad reciben pacientes principalmente de Ecuador, Aruba, Panamá, Estados Unidos, Alemania e Inglaterra. Para Norte de Santander puede ser una oportunidad de desarrollar este sector debido a su posición geoestratégica favorable por la cercanía a Venezuela y los países del Caribe.

\subsection{Cadena de Valor.}

"Para analizar las fuentes de la ventaja competitiva se necesita un medio sistemático que examine todas las actividades realizadas y la manera de interactuar" [16]. Para ello se establece la cadena de valor que consiste en dividir el sector en actividades estratégicamente relevantes a fin de entender su comportamiento, interacción, potencialidades y falencias. A continuación se enuncia la cadena de valor del sector de turismo de salud: (Ver Figura 2).
Enero - Junio 2016 ISSN 0122-820X E-ISSN 2422-5053 PP: 28-44 


\begin{tabular}{|c|c|c|c|c|}
\hline \multicolumn{5}{|c|}{$\begin{array}{l}\text { Infraestructura: Clínicas y Hospitales, Certificaciones Internacionales, zonas de atracción turística en la región, capacidad de } \\
\text { aceptar seguros internacionales y otras formas de financiación, Capacidad Hotelera. }\end{array}$} \\
\hline \multicolumn{5}{|c|}{$\begin{array}{l}\text { Gestión de Recursos Humanos: Disponibilidad de profesionales en salud, Universidades con programas de salud, nivel de } \\
\text { bilingüismo del personal asistencial y administrativo. }\end{array}$} \\
\hline \multicolumn{5}{|c|}{ Desarrollo Tecnológico: Telemedicina, Tecnología Médica. } \\
\hline \multicolumn{5}{|c|}{ Adquisiciones: Proveedores de suministros, medicamentos y equipos tecnológicos regionales y mundiales. } \\
\hline $\begin{array}{l}\text { Logística de } \\
\text { Entrada: } \\
-\quad \text { Servicios de } \\
\text { transporte. } \\
\text {-Agencias de turismo } \\
\text { médico }\end{array}$ & $\begin{array}{l}\text { Operaciones: } \\
\text { Procedimientos } \\
\text { médicos realizados } \\
\text { con estándares } \\
\text { internacionales. } \\
\text { Protocolos y guías de } \\
\text { manejo }\end{array}$ & $\begin{array}{l}\text { Logística de Salida. } \\
\text { Vuelos nacionales e } \\
\text { internacionales. } \\
\text { Sistema de } \\
\text { Transporte } \\
\text { medicalizado local }\end{array}$ & $\begin{array}{l}\text { Mercado técnica y } \\
\text { Ventas. } \\
\text { Precios } \\
\text { Agencias y paquetes } \\
\text { de turismo médico }\end{array}$ & $\begin{array}{l}\text { Servicios. } \\
\text { Seguimiento pos } \\
\text { operatorio en casa, } \\
\text { por otros médicos } \\
\text { contratados o a } \\
\text { través de } \\
\text { telemedicina }\end{array}$ \\
\hline
\end{tabular}

Figura 2. Cadena de valor de turismo de salud área metropolitana de Cúcuta Fuente: Autores

Infraestructura: Colombia cuenta con el ranking más alto de eficiencia en el sistema de salud [31]; tiene 16 de las mejores clínicas y hospitales de América latina [32], dos instituciones acreditadas por JCI y 22 más en trámite [20]. Las certificaciones internacionales y los médicos formados en el extranjero, que además hablan inglés, son factores que generan confianza a los pacientes extranjeros y se convierten en factores claves éxito en el sector.

El área metropolitana de Cúcuta, cuenta con 341 establecimientos en salud, de los cuales 56 son origen público y 285 de capital privado, tiene una disponibilidad de 889 camas hospitalarias, 294 de observación, 222 consultorios de consulta externa, 74 consultorios de servicio de urgencias, 68 mesas de parto, 124 unidades odontológicas y 12 salas de quirófanos [33].

En la actualidad se construye una clínica de cuarto nivel de complejidad y además se proyecta la construcción y dotación de 5 quirófanos y 30 camas UCI [34]. De estas instituciones 9 tienen sistema de gestión de calidad en procesos y ninguna tiene acreditación Icontec, JCI o JCAHO.
Los principales atractivos turísticos de la región son el Área Natural única de los Estoraques, Pamplona con sus templos religiosos y su alto nivel arquitectónico $\mathrm{y}$ cultural, el Parque Nacional CatatumboBarí, Parque Nacional del Tamá, el Templo Histórico de Villa del Rosario donde nació la República de Colombia, además de un gran legado histórico y cultural y una fuerte integración turística con Venezuela, lo que lo convierte en destino atractivo en el país [35], [36].

La capacidad de alojamiento es de 25 hoteles con 2.684 camas. Los principales servicios piscina, sauna, zona wifi, cambio de divisas, aire acondicionado, televisión por cable, gimnasio, spa, cajillas de seguridad, sala de negocios, servicios médicos, entre otros. En la actualidad se construye un hotel de talla mundial [36], [37].

Gestión de Recursos Humanos. Según el Ministerio de Comercio, Industria y Turismo [35] faltan recursos humanos capacitados en todas las profesiones universitarias relacionadas con la salud; los niveles de personal bilingüe son muy bajos; la percepción de riesgo de inseguridad aún es alta y como consecuencia será difícil, conseguir una 
acreditación internacional y por ende, atraer turistas de salud de países como Estados Unidos y Canadá.

En la región objeto de estudio tienen influencia especialmente las Universidades Francisco de Paula Santander, Universidad de Pamplona, Universidad de Santander, Universidad Simón Bolívar, Universidad Antonio Nariño y la Universidad Industrial de Santander. Todas ellas ofertan diferentes programas de salud, aunque ninguna ofrece Administración Turística y Hotelera, la cual es necesaria al menos para un actor clave del sector turismo de salud. La mayoría de las instituciones prestadoras de salud entrevistadas manifiestan que la ciudad cuenta con el personal médico idóneo y suficiente para atender pacientes de otros países; de hecho se atienden regularmente ya que hay muchos pacientes venezolanos que demandan servicios, a diario ya que esta zona está ubicada sobre la línea de frontera.

El estudio realizado en el Área Metropolitana, indica que el nivel de inglés o francés que tienen las instituciones para interactuar con los pacientes es: personal administrativo, malo; personal médico, regular; personal de enfermería y apoyo diagnóstico, malo. La mayoría de Instituciones Prestadoras de Salud entrevistadas manifiestan que la ciudad cuenta con el personal médico idóneo y suficiente para atender demanda interna y los pacientes que puedan llegar de otros países; de hecho se manifiestan que regularmente se atienden extranjeros ya que hay muchos pacientes venezolanos que demandan servicios, a diario.

\section{Desarrollo y Disponibilidad de Tecnología.}

El concepto de tecnología en salud hace referencia a cualquier medio que permita la aplicación del conocimiento científico en beneficio del cuidado de la salud y agrupa todos los medicamentos, dispositivos o procedimientos necesarios para promover la salud, prevenir, diagnosticar, tratar, rehabilitar o prestar cuidado paliativo a la enfermedad [38].

La industria de producción de tecnología médica es multimillonaria y se estima que alrededor del mundo existen 27.000 fabricantes de dispositivos médicos, dedicados a manufacturar cerca de 10.000 categorías diferentes de productos para el uso clínico y la salud. La industria de fabricación de los dispositivos médicos es liderada por Estados Unidos, no obstante, en los últimos años este liderazgo es desafiado por el instrumental producido en China e India, que en su gran mayoría es el resultado de los bajos costos en mano de obra [39].

Colombia también es productora de estas tecnologías contando con 888 empresas que abastecen el mercado local e internacional, ubicadas principalmente en Bogotá, Barranquilla, Caliy Medellín. Estas compañías aunque, exportan especialmente instrumentos y aparatos de medicina, ortopedia, masajes y terapias a países latinoamericanos y del Caribe, tan solo cubren el $10 \%$ de la demanda nacional [39]. Las compañías que proveen de materiales, tecnología e insumos en Norte de Santander son las mismas con presencia nacional, lo cual equipara los costos con otras regiones del país.

Adquisiciones. Colombia es el quinto mercado más grande en la región de medicamentos, por debajo de México, Brasil, Argentina y Venezuela. La industria nacional está dominada por multinacionales, siendo mayor en cantidad y valor de importaciones que las exportaciones [40]. Este país tiene los medicamentos más costosos del mundo [41], es evidente que los costos de la atención se vean influidos por este factor.

Logística de Entrada. La ubicación del Área Metropolitana de Cúcuta, es el nororiente de Colombia, en la frontera con Venezuela, dispone de un aeropuerto Internacional Camilo Daza, con vuelos regulares a Bogotá,
Enero - Junio 2016 ISSN 0122-820X E-ISSN 2422-5053 PP: 28-44 
No. 1

Enero - Junio 2016 ISSN 0122-820X E-ISSN 2422-5053
Medellín, Bucaramanga, Panamá [42] y otro aeropuerto en San Antonio, por el cual se comunica con el centro de Venezuela, el Caribe y Europa [43]. Posee tres vías terrestres principales que comunican con la Costa Atlántica a través de Ocaña, y por Bucaramanga con Bogotá, Medellín y la Costa Pacífica.

Por otra parte los actores que facilitan el desempeño de la industria del turismo en Colombia son el Ministerio de Salud, el Ministerio de Industria y Comercio, las Gobernaciones, Alcaldías, Clínicas públicas y privadas y los operadores, agencias de turismo de salud o facilitadores. Estos últimos tienen a cargo la promoción y el reclutamiento de los pacientes extranjeros, los cuales pueden ser propiedad del clúster en salud, de las clínicas y hospitales o como entidad de forma independiente que facilite $\mathrm{y}$ articule los servicios [24].

Operaciones. Los procedimientos médicos más solicitados por los turistas de salud se pueden clasificar en cuatro categorías, así: a) Cirugía mayor, este grupo incluyen procedimientos ortopédicos, reemplazos de rodilla y cadera, cirugía cardiaca, neurocirugía, procedimientos de cambio de sexo y cirugía bariátrica. b) Cirugía plástica o cosmética, que incluye procedimientos como mastopexia, abdominoplastia y el aumento de glúteos entre otros; c) Turismo dental, enfocado a la transformación de la sonrisa e incluyen el blanqueamiento dental, implantes, coronas, cirugía maxilofacial para corregir las lesiones y defectos en la cabeza, cuello, cara y mandíbula; y d) Tratamientos alternativos, por ejemplo terapias cancerosas, homeopatía, quiropráctica, neuropatía, terapia de mega vitaminas, hierbas y acupuntura. Los usuarios se caracterizan por ser personas sanas que buscan mantener o recibir ayuda a través de tratamientos de cuidados preventivos como exámenes médicos, la terapia de mega vitamina, el yoga y la meditación.
Según el Instituto de Departamental de Salud [44], en Norte de Santander, se encuentran habilitadas instituciones prestadoras de servicios con prácticamente todas estas especialidades, sin embargo ninguna de ellas cuenta con acreditación de alta calidad.

Logística de Salida. La recuperación del paciente, generalmente se hacen en la comodidad de un resort, acondicionado para garantizar el cuidado de la salud y el confort para el paciente y sus acompañantes [24]. El transporte entre las clínicas u hospitales y el hotel, se efectúa con ambulancias medicalizadas y el viaje a su lugar de origen puede hacerse en un vuelo comercial o chárter, $\mathrm{o}$ incluso en aviones ambulancia.

Mercadotecnia y Ventas. En Colombia, el turismo de salud hace parte de la Política de Mercadeo y Promoción Turística de Colombia denominada "Colombia Destino Turístico de Clase Mundial" [45], [46]. Mediante este mecanismo legal, se pueden establecer alianzas público-privadas que permitan promocionar el sector, a través de los medios masivos de comunicación y establecer convenios con Agencias de Turismo o facilitadores para que promociones y el recluten los pacientes extranjeros.

Servicios. Cuando el paciente regresa a su lugar de origen, puede ser monitoreado por el médico especialista mediante telemedicina, para lo cual deben establecerse acuerdos con algunas instituciones de salud del lugar de origen del paciente [47]. El concepto de telemedicina, telehealth o telecare, comprende el cuidado de la salud a distancia mediante la conexión entre la residencia del usuario y la persona que hace el seguimiento y control de su caso [48].

\subsection{La consolidación del Turismo de Salud}

En Colombia, el turismo de salud es parte del programa de transformación productiva realizado por Proexport, encabezado por el 
Ministerio de Comercio, Industria y Turismo, que busca potenciar sectores nuevos y emergentes con el fin de proyectarlos como de clase mundial, mediante la formulación y ejecución de planes de negocio sectoriales que favorezcan el crecimiento económico y el fortalecimiento del aparato productivo nacional a través de la alianza públicoempresarial [49].

El país se ha dado a la tarea de propiciar políticas que favorezcan el desarrollo empresarial, así mediante Documentos Conpes 3484 [50], "Política Nacional para la Transformación Productiva y la Promoción de las Micro, Pequeñas y Medianas Empresas: un esfuerzo público privado"; 3527 [51], "Política Nacional de Competitividad y Productividad"y 3582 [52], "Política Nacional de Ciencia, Tecnología e Innovación”, el Gobierno Nacional crea políticas que jalonen la transformación productiva a largo plazo impulsando diferentes sectores de la economía dentro de los cuales se encuentra el Turismo de Salud como eje fundamental de desarrollo económico y social del país. Los documentos Conpes, corresponden a las políticas públicas discutidas y aprobadas, por el Consejo Nacional de Política Económica y Social, y son los principales instrumentos de política pública en Colombia; a través de ellos se formalizan las decisiones gubernamentales tanto a nivel central como sectorial.

El plan de desarrollo del actual gobierno denominado "Prosperidad Para Todos" [53] incluye el turismo de salud como política de transformación productiva lo cual permite orientar esfuerzos públicos y privados, para que se consoliden o conviertan en sector de clase mundial, basado en desarrollo de capital humano, normativa y regulación, fortalecimiento de la industria y promoción e infraestructura.

En Norte de Santander, la Comisión Regional de Competitividad [54], plantea ocho objetivos estratégicos dentro de los que se cuenta el
Turismo, que permitan al Departamento ser competitivo y sostenible. La iniciativa plantea la aspiración de convertir el área metropolitana de Cúcuta, para el 2021, en pionero del turismo internacional de tránsito, con énfasis en el turismo histórico-cultural, el ecoturismo, y el agroturismo, aprovechando su ventaja de posición geoestratégica de frontera con Venezuela, diversidad de climas en un radio de 70 kilómetros y riqueza histórica teniendo en cuenta que aquí nació Colombia.

Los gobiernos de Norte de Santander y de la ciudad de Cúcuta, plantean la promoción de la región como destino turístico, a través de programas como la adopción de un plan de desarrollo turístico, la creación de un logo símbolo y slogan, campañas de formalización de los prestadores de servicios turísticos, campañas de creación y afianzamiento de la cultura turística en el sector hotelero, transporte y restaurantes; organización del clúster de turismo de salud, creación de una escuela de turismo en la región y mejoramiento, mantenimiento y conservación de los principales atractivos turísticos de la región.

En Norte de Santander, se han dado los primeros pasos para conformar un clúster en salud, integrado por 17 empresas entre las cuales se cuentan clínicas, laboratorios, centros especializados en estética, clínicas de medicina alternativa, clínicas oftalmológicas y odontológicas [55]. Estos esfuerzos apuntan a lograr que las instituciones de la región sean acreditadas, se mejore la infraestructura y capacidad tecnológica y se capacite al personal asistencial y administrativo.

\section{Conclusiones}

La búsqueda de asistencia médica fuera del país o región de origen es una práctica que gana importancia, y pacientes que desean tratamientos médicos más allá de sus fronteras provienen de países como Estados Unidos, Canadá y algunos de
Enero - Junio 2016 ISSN 0122-820X E-ISSN 2422-5053 PP: 28-44 
No. 1

Enero - Junio 2016 ISSN 0122-820X E-ISSN 2422-5053
Europa. Principalmente quienes solicitan este servicio son colombianos, residentes en esos países o personas que son originarias de esas regiones y que encuentran atractivos los precios, calidad, oportunidad, discreción y confidencialidad en sus tratamientos. Esta región tiene una posición geoestratégica favorable que debe aprovechar, en especial la cercanía con Venezuela y los países del Caribe, para fomentar el turismo médico en corto tiempo, debido al fácil acceso y a que las barreras culturales e idiomáticas no son mayores.

El turismo médico se convierten en una oportunidad para que familiares $y$ acompañantes disfruten de vacaciones $\mathrm{y}$ encuentren descanso y relajación visitando lugares cargados de belleza natural, histórica y cultural, como el Área Natural Única de los Estoraques, Pamplona con sus templos religiosos y su alto nivel arquitectónico y cultural, el Parque Nacional CatatumboBarí, Parque Nacional del Tamá, el Templo Histórico de Villa del Rosario donde nació la República de Colombia y la frontera venezolana que siempre es un destino turístico y comercial.

Los estándares de acreditación son un factor vital en la evaluación de la calidad de la atención de los hospitales extranjeros y las agencias de intermediación. Es por eso que la acreditación y reconocimiento de las IPS por instituciones acreditadoras de alta calidad como Joint Commission International, es la mayor dificultad y al mismo tiempo el mayor reto que tiene el sector para lograr tener características de clase mundial.

Otra debilidad del sector, es el bajo nivel de bilingüismo, y como consecuencia será difícil atraer turistas de salud de países como Estados Unidos y Canadá, donde se encuentra la mayor concentración de los potenciales clientes; por lo que será necesario establecer programas de entrenamiento que logren un interacción natural con el paciente y el diligenciamiento de la historia clínica en su idioma natal.

Las especialidades médicas solicitadas por los turistas médicos son cirugía plástica, procedimientos de cambio de sexo o tratamientos relacionados con rehabilitación por drogadicción, medicina preventiva, procedimientos menores en cirugía estética y dental, procedimientos invasivos mayores como cirugía a corazón abierto, reemplazo de rodilla o cadera, columna vertebral y cirugía bariátrica. Todas estas especialidades están habilitadas en la región, por las respectivas autoridades de salud, aunque ninguna de ellas está acreditada por organismos internacionales. Los Gobiernos departamental y nacional impulsan proyectos para lograr que las instituciones de salud obtengan ese reconocimiento.

La venta de los servicios a pacientes de otros países, se basa principalmente en el diferencial de precios, que para Colombia pueden representar un ahorro entre el 10\% y el $40 \%$, pero es necesario combinarlo con el "esencialismo estratégico", y aprovechar la belleza natural de los paisajes, riqueza histórica, diversidad cultural, posición geoestratégica, diversidad de climas en un radio de 70 kilómetros y riqueza histórica teniendo en cuenta que aquí nació esta república.

\section{Referencias}

[1] Superintendencia Nacional de Salud. Instituciones prestadoras de servicios de salud, Norte de Santander, 02013. [Online]. Disponible en: http://www. supersalud.gov.co/mapaNteSantander. html.

[2] D. A. Shaywitz y D.A. Ausiello, "Global Health: A Chance for Western Physicians to give - and Receive", The American Journal of Medicine, vol. 113, no. 4, pp. 354-357, 2002. 
[3] Deloitte Development LLC. Medical Tourism: Consumers in Search of Value. 2008. [En linea]. Disponible en: https://www.academia.edu/9144718/ Medical_Tourism_Consumers_in Search_of_Value_Produced_by_the _ Deloitte_Center_for_Health_Solutions.

[4] M. Ricafort. "A study of influencing factors that lead Medical tourists to choose Thailand Hospitals as medical tourism destination". Tesis de Magister, Webster University, Cha-am, Thailand, 2011.

[5] F. Melgosa. Turismo de salud: Termalismo y balnearios", en la obra colectiva "III Congreso de turismo universidad y empresa", dirigido por BLANQUER CRIADO, D., Ed. Tirant lo Blanch, pp. 359-386, 2000.

[6] M. Bolis. El turismo de salud en América Latina y el Caribe de habla inglesa. 54 Congreso de la Federación Mundial de Termalismo y Climatología (FEMTEC), II Congreso Latinoamericano de Turismo y Salud (FTL), III Congreso Internacional de Turismo y Salud, Varadero, Cuba, 1923 de noviembre de 2001.

[7] B. Adriaensen, "El debate postcolonial en América Latina: la recepción de Transculturación narrativa de Ángel Rama". Congreso del Instituto Internacional de Literatura Iberoamericana, edición 33. Salamanca, Universidad de Salamanca, pp. 26-30, junio de 2000.

[8] W. Gesler, "Lourdes: healing in a place of pilgrimage", Health \& Place, vol. 2, no. 2, pp. 95-105, 1996.

[9] A. Williams, "Spiritual therapeutic landscapes and healing: a case study of St. Anne de Beaupre, Quebec, Canada",
Social Science \& Medicine. vol. 70, no. 10, pp. 1633-1640, 2010.

[10] F. Smyth, "Medical geography: therapeutic places, spaces and networks", Progress in Human Geography, vol. 29, no. 4, pp. 488-495, 2005.

[11] A. Williams, "Therapeutic landscapes and holistic medicine", Social Science \& Medicine, vol. 46, no. 9 pp. $1193-$ 1203, 1998.

[12] L. Turner, "First world health care at third world prices: globalization, bioethics and medical tourism", BioSocieties, vol. 2, no. 3, pp. 303-325, 2007.

[13] R. Guha, y G. Spivak. "Subaltern studies: deconstructing historiography". En Selected subaltern studies. New York: Oxford University Press, 1988.

[14] K. Nkrumah. "Neocolonialism: The last stage of imperialism". New York: International Publishers, 1966.

[15] I. Dyck y R. Kearns, "Transforming the relations of research: towards culturally safe geographies of health and healing", Health \& Place, vol. 1, no. 3, pp. 137147, 1995.

[16] M. Porter. "Ventaja Competitiva. Creación y Sostenimiento de un Desempeño Superior". México, Patria, 2013.

[17] McKinsey \& Company. Mapping the market for medical travel. 2008. [En linea]. Disponible en: http://www. heal-wheel-india.com/white-pappers/ McKinsey-Report-Medical-Travel.pdf.

[18] P. Smith y D. Forgione. "Global Outsourcing of Healthcare: A Medical Tourism Decision Model", Journal
Enero - Junio 2016 ISSN 0122-820X E-ISSN 2422-5053 PP: 28-44 
No. 1

Enero - Junio 2016 ISSN $0122-820 \mathrm{X}$ E-ISSN 2422-5053 PP: 28-44

[19] Fundación para la Acreditación y DesarrolloAsistenciasl(FADA). Boletín Informativo, vol. 9, pp. 1-5, septiembre de 2014. [En linea]. Disponible en: http://www.acreditacionfada.org/ uploads/documentosJCI/Boletin_JCI_ FADA_9_septiembre_2014.pdf.

[20] Proexport Colombia. Comprender las oportunidades de la industria de la salud en Colombia. 2012. [En linea]. Disponible en: http://www. procolombia.co/sites/default/files/ Presentacion_del_sector.pdf.

[21] M. Wongkit, B. McKercher. "Toward a typology of medical tourists: A case study of Thailand", Tourism Management. vol. 38, pp. 4-12, 2013.

[22] J. Connell, "Medical tourism: sea, sun, sand and surgery", Tourism Management, vol. 27, no. 6, pp. 10931100, 2006.

[23] D. Horowitz, J. Rosensweig, y C. Jones, "Medical tourism: globalization of the Healthcare Marketplace", Medscape General Medicine, vol. 9, no. 4, pp. 1-25, 2007.

[24] J. Syed, et al. "Investigating Critical Success Factors Of Value Chain In
Entrant Perspective", Tesis de Magister, Tanaka Business School Imperial College. Londres, Inglaterra, 2008.

[27] N. MacReady, "Developing countries court medical tourists", The Lancet, vol. 369, no. 9576, pp. 1849-1850, 2007.

[28] P. Gahlinger. "The Medical Tourism Travel Guide: Your Complete Reference to Top-Quality, Low-Cost Dental, Cosmetic, Medical Care \& Surgery Overseas". North Branch, Sunrise River Press, 2008.

[29] S. Mitra, Medical Tourism: The Way To Go. [En linea]. 2007. Disponible en: http://www.frost. com/prod/servlet/market-insight-top. pag?docid $=108452141$.

[30] A. NaRanong y V. NaRanong, "The effects of medical tourism: Thailand's experience", Bulletin of the World Health Organization, vol. 89, no. 5, pp. 336-344, 2011.

[31] Organización Mundial De La Salud OMS. The World Health Report. [En linea]. Disponible en: http://www.who. int/whr/2000/en/whr00_en.pdf.

[32] América Económica. Rankin de Clínicas y Hospitales 2012. [En linea]. Disponible en: http://rankings. americaeconomia.com/2012/clinicas-yhospitales/ranking.php.

[33] Instituto Departamental de Salud. Análisis de situación de salud (ASIS) en municipios de frontera. 2010. [En linea]. Disponible en: https://www. minsalud.gov.co/plandecenal/mapa/ Analisis-de-Situacion-de-Salud-enmunicipios-de-frontera-DepartamentoNorte-Santander.pdf.

[34] Gobernación Norte de Santander. "Plan de Desarrollo para Norte de Santander.

[26] J. Shah, "An Insight into Malaysia's Medical Tourism Industry from New 
2012-2015: Un Norte Pa'lante”. Cúcuta, Colombia, pp. 1-179, 2012.

[35] Ministerio de Comercio, Industria y Turismo. Desarrollando sectores de clase mundial en Colombia sector de turismo de salud. Resumen Ejecutivo. Bogotá: Diario oficial. 2009. [En linea]. Disponible en: https://econotur. files.wordpress.com/2013/02/ptp20090709-resumen-ejecutivo-turismode-salud.pdf.

[36] Departamento Nacional de Planeación. "Agenda Interna para la Productividad y la Competitividad. Documento regional, Norte de Santander”. Bogotá, Colombia, 2007.

[37] Cotelco. Capacidad de Alojamiento en el Área Metropolitana de Cúcuta. Información solicitada por el autor con el fin de desarrollar este estudio. Cúcuta, Colombia. 2014.

[38] Comisión de regulación en salud. Acuerdo No 003 de 2009 [En linea]. Disponible en: http://www.ilo.org/dyn/ travail/docs/1500/ACUERDO $\% 20$ 003\%20DE\%202009.pdf.

[39] E. Mateus. "La Saludable Industria de los Dispositivos Médicos" Revista Metalactual. no. 28, pp. 68-72, mayojulio 2013.

[40] J. Vásquez, K. Portilla y S. Acosta, "Regulación en el mercado farmacéutico colombiano", Revista de Ciencias Sociales (Ve), vol. 16, no. 2, pp. 197209, 2010.

[41] Health Action International. Estar enfermo y necesitar medicamentos, puede ser una costosa desgracia en muchos países. Un recorrido en un día por el precio de un medicamento en 93 países. 2009. [En linea]. Disponible en: http://www.haiweb.
org/medicineprices/05012010/Global_ briefing_note_Spanish.pdf.

[42] Aeronáutica Civil. Estadísticas Operacionales. 2014. [En linea]. Disponible en: http://www.aerocivil. gov.co/AAeronautica/Estadisticas/ TAereo/EOperacionales/Paginas/ EOperacionales.aspx

[43] Aeropuerto de San Antonio del Táchira, Juan Vicente Gómez. Estadísticas detalladas aeropuerto de San Antonio del Táchira (San Antonio del Táchira). 2014. [En linea]. Disponible en: http:// www.europelowcost.es/aeropuertos/ san-antonio-del-tachira-juan-vicentegomez-svz.aspx.

[44] Instituto Departamental de Salud de Norte de Santander. Instituciones Prestadoras de Salud y Especialidades Médicas en Norte de Santander. Información suministrada por la entidad al autor con el propósito de realizar este estudio. Cúcuta, Norte de Santander. 2013.

[45] Ministerio de Comercio, Industria y Turismo. "Política de Mercadeo y Promoción Turística de Colombia denominada: Colombia Destino Turístico de Clase Mundial”. Bogotá, Colombia, Diario oficial, 2011.

[46] Ministerio de Comercio, Industria y Turismo. "Desarrollando sectores de clase mundial en Colombia sector de turismo de salud". Resumen Ejecutivo. Bogotá, Colombia, Diario oficial, 2009.

[47] K. Cabezas, M. Hernández, et al. Medical Tourism Emerging Cluster. Universidad Popular Autónoma del Estado de Puebla. Puebla, México, 2012.

[48] S. Litewka, "Telemedicina: Un desafío para América latina", Acta 
No. 1

Enero - Junio 2016

ISSN 0122-820X

E-ISSN 2422-5053

PP: $28-44$

[49] A. Barriga, M. Farías, A. Ruiz, A. Sánchez, W. Jiménez, et al. "Turismo en salud: una tendencia mundial que se abre paso en Colombia", Ciencia y Tecnología en Salud oral y visual, vol. 9, no.1, pp. 125-137, 2011.

[50] República de Colombia. Política Nacional para la transformación productiva y la promoción de las micro, pequeñas y medianas empresas: un esfuerzo público-privado. Documento Conpes No. 3484. 2007. [En linea]. Disponible en: http://www.huila.gov.co/ documentos/C/CONPES3484de2007. pdf.

[51] República de Colombia. Política Nacional de Competitividad y Productividad. Documento Conpes 3527. 2008. [En linea]. Disponible

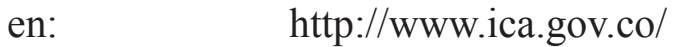
getattachment/9ead52fd-f432-4175b42a-484ea0662194/2008CN3527. aspx.

[52] República de Colombia. Política Nacional de Ciencia, Tecnología e Innovación. Documento Conpes 3582. 2009. [En linea]. Disponible en: http://www.colciencias.gov.co/sites/ default/files/upload/reglamentacion/ conpes_3582.pdf.

44

[53] Departamento Nacional de Planeación. "Plan Nacional de Desarrollo, 20102014". 2011. [En linea]. Disponible en: https://colaboracion.dnp.gov.co/CDT/ PND/PND2010-2014\%20Tomo\%20 I\%20CD.pdf.

[54] Comisión Regional de Competitividad. El Plan Regional de Competitividad de Norte de Santander. 2007.[En linea].
Disponible en: www.mincit.gov.co/ descargar.php?id=63523.

[55] Cámara de Comercio de Cúcuta. Clúster en Salud en Norte de Santander. Información solicitada por el autor con el fin de desarrollar este estudio. Cúcuta, Colombia, 2013. 